\title{
It's all about the spaces between cells: role of extracellular matrix in liver fibrosis
}

\author{
Amit Khurana ${ }^{1,2}$, Nilofer Sayed ${ }^{3}$, Prince Allawadhi $^{4}$, Ralf Weiskirchen $^{2} \wedge$ \\ ${ }^{1}$ Center for Biomedical Engineering (CBME), Indian Institute of Technology (IIT), Hauz Khas, New Delhi, India; ${ }^{2}$ Institute of Molecular \\ Pathobiochemistry, Experimental Gene Therapy and Clinical Chemistry (IFMPEGKC), RWTH University Hospital Aachen, Aachen, Germany; \\ ${ }^{3}$ Department of Veterinary Pharmacology and Toxicology, College of Veterinary Science, Rajendranagar, Hyderabad, Telangana, India; ${ }^{4}$ Department \\ of Biotechnology, Indian Institute of Technology (IIT), Roorkee, Uttarakhand, India \\ Contributions: (I) Conception and design: A Khurana, R Weiskirchen; (II) Administrative support: None; (III) Provision of study materials or patients: \\ None; (IV) Collection and assembly of data: A Khurana; (V) Data analysis and interpretation: None; (VI) Manuscript writing: All authors; (VII) Final \\ approval of manuscript: All authors. \\ Correspondence to: Amit Khurana. Center for Biomedical Engineering (CBME), Indian Institute of Technology (IIT), Hauz Khas, New Delhi, India; \\ Institute of Molecular Pathobiochemistry, Experimental Gene Therapy and Clinical Chemistry (IFMPEGKC), RWTH University Hospital Aachen, \\ Aachen, Germany. Email: ak3.khurana@gmail.com; Ralf Weiskirchen. Institute of Molecular Pathobiochemistry, Experimental Gene Therapy and \\ Clinical Chemistry (IFMPEGKC), RWTH University Hospital Aachen, Aachen, Germany. Email: rweiskirchen@ukaachen.de.
}

\begin{abstract}
Liver fibrosis is one of the leading complications of a variety of chronic liver disorders, including the nonalcoholic fatty liver disease, nonalcoholic steatohepatitis, liver cirrhosis and liver failure. The progression of liver fibrosis is driven by chronic inflammation, which activates the secretory fibroblasts to the myofibroblast phenotype. These specialized liver cells are called as hepatic stellate cells (HSCs). The excessive extracellular matrix (ECM) secretion creates a large number of complications. Fibrosis is the result of imbalance between the matrix synthesizing and matrix degrading factors. The major ECM proteins include the matrix metalloproteinases (MMPs), tissue inhibitor of metalloproteinases (TIMPs), lysyl oxidases (LOX), lysyl oxidase-like (LOXLs) enzymes, tenascins and others. These ECM proteins present novel avenues for the therapeutics of liver fibrosis. The current review highlights the major role played by these critical matrix proteins in liver fibrosis. Further, some of the targeted formulations used against these proteins are discussed and suggestions are provided to select the course of research for successful clinical translation of basic research findings for the amelioration of liver fibrosis.
\end{abstract}

Keywords: Liver fibrosis; extracellular matrix; matrix metalloproteinases (MMPs); tissue inhibitor of metalloproteinases (TIMPs); lysyl oxidases (LOX)

Submitted Mar 28, 2020. Accepted for publication Jul 22, 2020.

doi: 10.21037/atm-20-2948

View this article at: http://dx.doi.org/10.21037/atm-20-2948

\section{Introduction}

Fibrosis is an integral part of the normal wound healing process. However, a recurrent insult to the tissues in the form of chronic inflammation leads to aberration in the wound healing process. This hampers the resolution of scar and uncontrolled fibrotic signaling overrules the resolution potential of enzymes leading to consistent replacement of the parenchymal tissue by the extracellular matrix (ECM) proteins (1). The potential causes of chronic liver injury are hepatitis $\mathrm{B}$ and hepatitis $\mathrm{C}$ viral infections, chronic exposure to alcohol and toxins in the form of drugs and environmental pollutants, diseases such as non-alcoholic steatohepatitis (NASH) and cholangitis (2). Untreated liver fibrosis initially causes portal hypertension, ascites and

^ ORCID: 0000-0003-3888-0931. 
bleeding from the esophageal varices. Later, progression takes the route of liver cirrhosis, finally, ending in the form of hepatocellular carcinoma, which has a poor outcome and a high mortality rate. Despite awareness and wide research done in terms of the factors and mechanisms involved in liver fibrosis, clinical translation of potential strategies is lagging. A recent report suggested that if the trend continues, then cirrhosis will be the $12^{\text {th }}$ leading cause of death by 2020 (3).

Hepatic parenchyma is made up of epithelial cells (hepatocytes), endothelial cells, resident nonparenchymal cells, including hepatic stellate cells (HSCs) and Kupffer cells $(\mathrm{KC})$. The sinusoid is the hepatic microvascular unit. It has an endothelial lining distinguished by fenestration of pores and is separated from hepatocytes by subendothelial space of Disse where HSCs reside (4). The ECM provides structural integrity to the cells within the tissue compartment, it triggers the polarization of cells and also determines the fate of gene expression (5). The ECM can be distinguished into three fractions depending on its position; one is the pericellular matrix located in close connection with cell membrane components, second is the part of the matrix filling up the interstitial spaces, and the third remainder parts of the basement membrane supporting the epithelial, endothelial and mesenchymal cells (6). Liver fibrosis is characterized by excessive accumulation of interstitial or fibrillar ECM.

In this review, we discuss the advances in the understanding of the physiological role of the ECM and the factors causing aberrations in the quality and quantity of ECM. The role of important ECM components and various novel strategies are reviewed for understanding how new findings can be harnessed for the therapy of liver fibrosis.

\section{Dysregulation of extracellular matrix synthesis: key to fibrosis}

Physiologically, the ECM is a dynamic scaffold that maintains the cells in a three-dimensional network mainly composed of proteoglycans, fibrous proteins and range of enzymes (7). The proteoglycans are the major component providing the hydrated gel consistency to the ECM, modulating cell adhesion and sustain high osmotic pressure. Proteoglycans contain glycosaminoglycan chains covalently linked to proteins. These chains can be further classified based on their sulphation pattern into sulphated glycosaminoglycans such as chondroitin, heparan and keratan sulfate; whereas the important non-sulphated glycosaminoglycan is hyaluronic acid (8). Recent studies have shown that a novel proteoglycan, lumican, which plays a role in fibrillogenesis in extrahepatic tissues is upregulated in hepatitis $\mathrm{C}$-infected clinical samples and in rodents suffering from acute and chronic liver injury, thereby eliciting its importance as a target in the mitigation of fibrosis or as a potential diagnostic marker (9). Glycoproteins that make up the ECM are fibronectin, which is responsible for interaction between collagen and heparin. It acts as a chemoattractant and is also a growth factor for mesenchymal cells (10). The epithelial-to-mesenchymaltransition (EMT) is a fundamental process during which the fibroblasts change their phenotype to highly motile and excessive ECM secreting myofibroblasts. Hence, an upregulation of fibronectin is observed during EMT. Fibronectin expression correlates with the surge in the levels of transforming growth factor $\beta-1$ (TGF- $\beta 1$ ) and to influence collagen deposition thereby suggesting the role of fibronectin in liver injury (11). Fibronectin is of two types; cellular fibronectin ( $\mathrm{cFn}$ ) and plasma fibronectin which are found in balanced levels during normal physiological functioning. $\mathrm{cFn}$ has been found to increase in proportion to the extent of tissue injury. Excessive cFn deposition prepares the stage for the collagenous accumulation, mainly type I and III collagen within the matrix. Fibronectin stretched by cellular traction exposes the hidden integrin binding sites thereby stimulating collagen gel contraction through cell-fibronectin binding integrin, $\alpha 5 \beta 1$.

Vitronectin is another adhesion molecule in the ECM, involved in binding of collagen, elastin and proteoglycans within the ECM (12). Vitronectin is an indicator of the chronicity of the injury (13). Laminin forms the main component of the basement membrane and interacts with type IV collagen, heparan sulfate, proteoglycan and entactin. Entactin is bound centrally to laminin. Undulin is found in association with mature collagen fibrils I and III (14). Cell adhesion to ECM is mediated by ECM receptors namely integrins, discoidin domain and syndecans. Syndecan-1 is normally expressed on the surface of hepatocytes and cholangiocytes, which increases in liver diseases and representing a good molecular marker for liver fibrosis induced by hepatitis $\mathrm{C}$ infection (15).

Decorin is a leucine-rich proteoglycan found in close association with TGF- $\beta$, one of the vital pro-inflammatory cytokines to initiate fibrogenesis. It was reported that decorin is protective in nature by inhibiting the overactivity of this cytokine. It not only affects TGF- $\beta$ functioning, but also regulates the expression of other ECM components such as fibronectin and collagenase activity (16). 


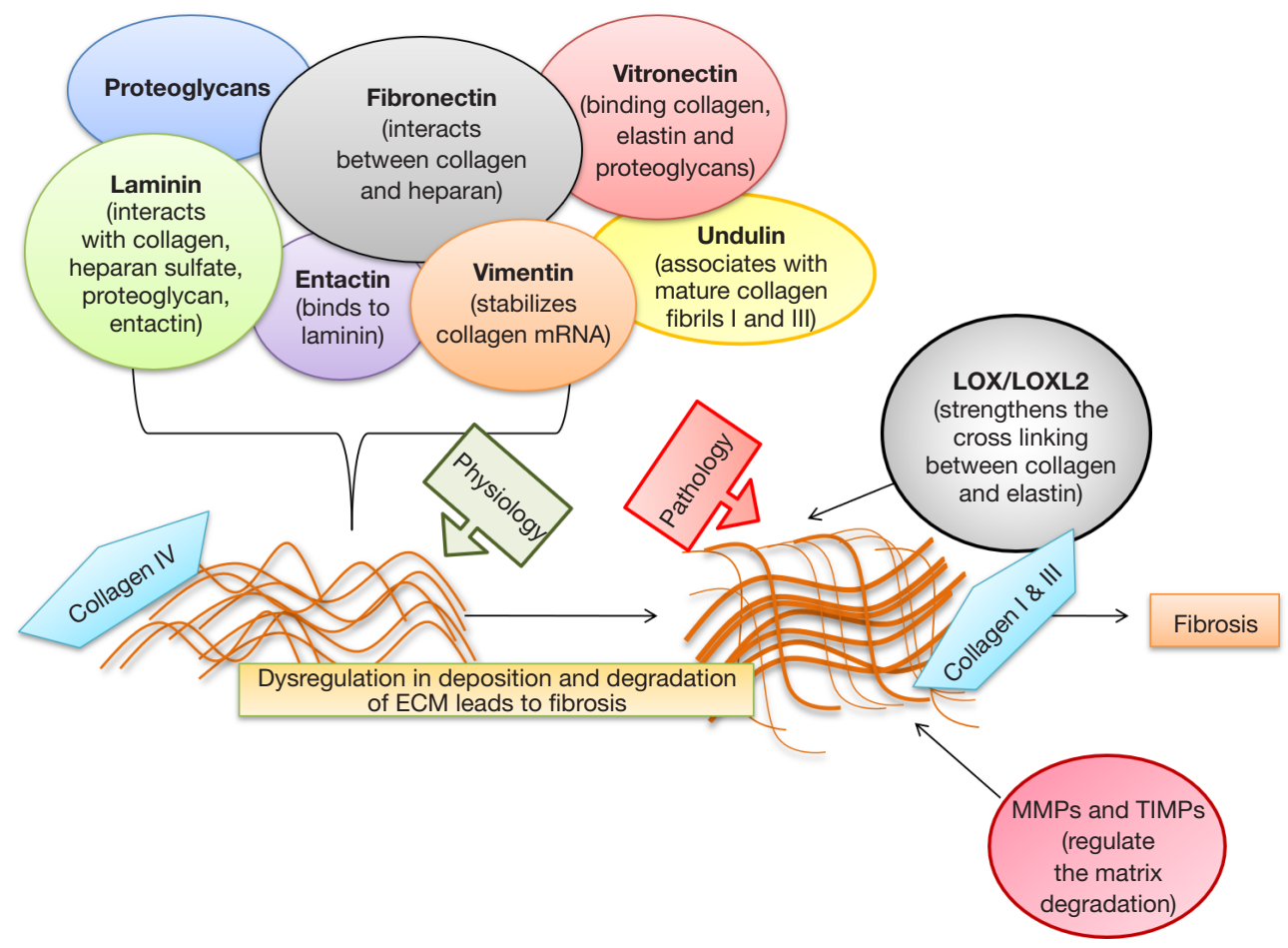

Figure 1 The role of various extracellular matrix proteins in the pathogenesis of liver fibrosis. Individual activities of proteoglycans and enzymes are depicted. Under physiological conditions, the concert of these proteins guarantees a balanced synthesis of extracellular matrix (ECM). However, under pathogenetic conditions, it comes to overshooting reactions resulting in dysregulation in deposition and degradation of ECM, finally resulting in fibrosis. LO, lysyl oxidase(s); LOXL2, lysyl oxidase homolog 2.

After liver injury, disruption of matrix and deposition by fibrillar collagens, composed of collagen I, III and fibronectin, occurs. These quantitative and qualitative changes in ECM alter the matrix microenvironment. It also incorporates a range of growth factors and ECM enzymes. Thus, deposition of ECM results in the loss of normal fenestrations which provokes impairment of normal bidirectional metabolic exchange between portal venous flow and hepatocytes leading to further progression of the disease. The chronic fibrogenic state eventually leads to cirrhosis characterized by distortion of liver parenchyma and vascular architecture. Reactive oxygen species (ROS) cause oxidative stress in hepatic cells, KCs, HSCs, natural killer cells (NK), lymphocytes and dendritic cells. The dendritic cells release various cytokines and interleukins (IL-1, IL-6), interferons (IFN- $\alpha$, IFN- $\gamma$ ) augmenting the inflammatory cascade. This initiates TGF- $\beta$-driven fibrogenic signaling, which transforms the quiescent HSCs to proliferative, contractile and highly fibrogenic myofibroblast cell type, which in turn leads to excess ECM production and acting as a positive feedback for the incorporation of more growth factors. This sequence of events ultimately leads to liver fibrosis (17).

Vimentin is a member of type III intermediate filaments and a reliable marker for cells of mesenchymal origin. Vimentin filaments are involved in motility, maintenance of cell shape and endurance of mechanical stress in mesenchymal cells. During activation of HSCs, there is a 16 -fold prolongation of half-life of collagen- $\alpha 1$ (I) mRNA, which is responsible for a 50 -fold increase in its expression. Thus, the transformation of fibroblasts into myofibroblasts is associated with increased stability of collagen- $\alpha 1$ (I) mRNA. Vimentin has a major role in stabilization of this collagen mRNA hence, vimentin inhibition could be an attractive strategy for the mitigation of liver fibrosis (18). Figure 1 depicts the representation of major matrix proteins which are involved in the pathogenesis of liver fibrosis.

\section{Matrix enzymes and their role in liver fibrosis}

Matrix metalloproteinases (MMPs), tissue inhibitor of metalloproteinases (TIMPs), lysyl oxidase (LOX) and lysyl 
oxidase like (LOXLs) enzymes are the major enzymes regulating the ECM. These modulate the progression and resolution of fibrosis. Tissue injury leads to apoptosis and necrosis of cells, which stimulates the antifibrinolytic coagulation factors and platelet activation. This phase is also accompanied by the induction of profibrogenic MMPs such as MMP-2 and MMP-9 disrupting the basal membrane and stimulating the production of fibrillar collagen. Further, there is release of inflammatory mediators. In addition, macrophages, neutrophils and leukocytes lead to increased expression and release of platelet-derived growth factor (PDGF), TGF- $\beta$ and IL-13 playing key roles in the transition of HSCs to activated myofibroblasts (19).

MMPs are zinc-dependent endopeptidases. There are 23 membrane-bound members in this enzyme family, which are commonly synthesized as latent zymogen forms. They are mainly involved in the degradation of collagen, however all MMPs are not antifibrotic. Physiologically the basement membrane is comprised of collagen IV but this is progressively replaced by collagen I and III secreted by activated HSCs bringing about the stiffness of the fibrotic tissue. MMP2, also called gelatinase A, is involved in HSC proliferation and also plays a role in the activation of TGF- $\beta$. MMP2 is responsible for the mitogenic proliferation and invasive nature of HSCs. ROS in HSCs mediate the upregulation of MMP2 expression via extracellular signal-regulated kinases $1 / 2$ (ERK1/2) and phosphoinositide 3-kinase (PI3K) signaling mechanism. In the presence of collagen I, activated HSCs secrete MMP2, further degrading collagen IV, which is normally present in liver and is substituted with collagen I and III. MMP9 is secreted by KCs. This proenzyme form is activated by plasmin and stromelysin. Similar to MMP2, this proteinase is also involved in the degradation of the normal collagen matrix. In addition, MMP 3 converts latent TGF- $\beta$ to active state, but there is no clear data available to confirm its role.

Thus, MMP2, MMP9 and MMP3 are highly expressed during the acute phase of tissue injury. The MMPs, which are antifibrotic in nature, include MMP1, MMP8 and MMP19. MMP1 induces the expression of collagenase enabling degradation of the fibrotic scar. MMP8 cleaves interstitial collagen I and III. MMP19 is found to degrade ECM. This enzyme is profibrotic in early phases after injury and antifibrotic during the resolution phase (20). ECM function and physiology is tightly regulated by MMPs. These are secreted in an inactive form and carry zinc at their catalytic site $(21,22)$. To facilitate cell migration and deposition of new ECM compounds, tissue MMPs degrade different
ECM substances such as fibrin, cartilage, tendon, and other. All MMPs have several common domains and relatively similar structure. MMPs contain 4 main domains. The first one possesses the signaling peptide targeting the secreting molecule which is present in an inactive form. The second domain is the propeptide domain that must be cleaved to release the active form. The third domain contains the zincbinding catalytic domain. Being structurally similar to the hemopexin, this domain is often referred to the hemopexin domain. Structural differences in this domain decide about the specificity of individual MMPs for a particular substrate. Activation of MMPs is regulated at three levels, firstly at gene level (transcriptional control), second at the molecular level including factors relevant in converting the proenzyme (inactive from) to its active form and lastly by MMPs inhibitors. MMPs are highly active at the site of injury for fast repair and healing. Sometimes their hyperactivity makes the condition pathological, which may culminate as bone resorption, liver fibrosis, pancreatitis, rheumatoid arthritis, atherosclerosis and intense inflammation $(22,23)$.

LOX and LOXL2 are lysyl tyrosylquinone- and copperdependent amine oxidase enzymes catalyzing the covalent cross-linking of collagen and elastin. LOXL2 is found on the surfaces of endothelial cell-derived exosomes. This facilitates the cross-linking of collagen and elastin fibers through selective oxidative deamination of lysine residues, thereby imparting stiffness to the hepatic parenchyma. Targeting LOXL2 is one of the most promising approach towards the reversal of fibrosis. Moreover, LOXL2 is also shown to interact with histones and transcription of Snail, leading to suppression of E-cadherin gene expression and promotion of EMT transition. Snail is a repressor gene and EMT inducer. LOXL2 is a co-repressor and controls cadherin 1 (CDH1) gene transcription and selectively regulates $\mathrm{H} 3 \mathrm{~K} 4 \mathrm{me} 3$ deamination. $\mathrm{H} 3 \mathrm{~K} 4 \mathrm{me} 3$ oxidation on the other hand could affect the expression of LOXL2.

The acute tissue insult activates these cells, leading to EMT and formation of myofibroblasts, triggering an increased expression and deposition of ECM compounds (24). Thus, the hepatic parenchyma hosting a variety of parenchymal and non-parenchymal cells, proteoglycans and glycoproteins along with ECM enzymes, namely LOXL, MMPs and TIMPs all play an integral role in the development and resolution of fibrosis.

\section{Tenascin $\mathbf{C}$ and its role in liver fibrosis}

TenascinC belongs to the family of tenascins proteins, 
typically having a six-arms-like appearance, which comprise four types, namely tenascin C, R, W2 and X (25). Tenascin $\mathrm{C}$ is most prominently explored among its family for its physiological and pathological role (26). Its hexameric arms like structure are linked by disulfide bonding forming a molecule of $2,000 \mathrm{kDa}$ in size. It can bind to fibronectin forming a mesh-like network in ECM and has a partial DNA sequence resemblance with Col-XIV/undulin and fibronectin (27). It is synthesized mainly in the nervous system and connective tissues, but it is distributed in the different sites and conditions of the body like wounds, tumors, ligament, embryonic tissues, skeletal system, tendon and many more (28).

Tenascin $\mathrm{C}$ has been found to be involved in regulating the activity of different biochemical entities, especially epidermal growth factor and MMPs. It stimulates the osteoblast function indicating its role in bone formation and metabolism during fracture (29).

Normally tenascin-C is not present in adult liver and it is expected to be a prominent damage-associated molecular pattern (DAMP) protein inside the ECM. Gene deletion of tenascin $\mathrm{C}$ resulted in reduced recruitment of leukocyte and lower expression of cytokines after hepatic reperfusion (30). Tenascin $\mathrm{C}$ is reported to be found mainly in the fat storing cells and is one of the typical components in ECM formation (31). It is widely explored in the area of fibrosis and its role in liver fibrosis progression is well reported in various experimental studies (32). It is further involved in modulation of ECM activity. In normal condition, ECM is involved in regulating the function and morphology of epithelial, mesenchymal cells, cell migration and cell attachment. In contrast, in altered condition it deviates from normal physiological function that results in different pathological conditions out of which liver fibrosis is one of the major outcomes $(29,33)$. The level of tenascin C was found to be profoundly over expressed in the bile duct ligation model of experimental liver fibrosis. After day 2 and 3, tenascin $C$ was more deeply expressed in between proliferating ductules at the margin portal tracts and surrounding interlobular bile ducts. Similarly on day 7 and 14, tenascin was still highly intense at the interface in between portal tract and liver parenchyma. Tenascin distribution was localized and immensely expressed at connective tissue-parenchymal interface suggesting tenascin as a key player involved in normal functioning of the ECM, while elevated quantities provoke the development of fibrotic condition (34). In response to any injury to the liver, the first changes include the synthesis of new
ECM proteins, which appear very early and tenascin C is one of those prominently expressed proteins. If higher levels remain during phases of prolonged injury, then it contributes to the replacement of normal liver parenchymal tissue by fibrotic tissue (21). It is evident from various animal studies that tenascin $\mathrm{C}$ strongly contributes to the development of fibrosis in various disease conditions including tumor, inflammation, atherosclerosis and liver fibrosis. It is further assumed that tenascin $\mathrm{C}$ upholds the fibrotic environment by stimulating inflammatory mediators like cytokine (e.g., TGF- $\beta$ ) and by recruiting HSCs $(35,36)$. To confirm the role of the tenascin $\mathrm{C}$ in liver fibrogenesis, a comparative study on tenascin C-knock out (TNKO) mice and wild type mice was carried out (21). The liver immunohistochemistry revealed a gradual rise in the levels of tenascin $\mathrm{C}$ and the quantities of mRNA coding for tenascin $\mathrm{C}$ were also augmented in wild type mice during fibrogenesis. In contrast, in TNKO mice only negligible changes were observed, indicating an important role of tenascin $\mathrm{C}$ in liver fibrosis. Therefore, attenuation of tenascin $\mathrm{C}$ expression could be a novel strategy to treat liver fibrosis (37). Important pathological functions of tenascins in liver fibrogenesis are depicted in Figure 2.

\section{Role of extracellular enzymes in healing and repair}

The body's reaction to early tissue damage is both complex and very coordinated. This reaction starts with coagulation (hemostasis), inflammation, proliferation (expansion), and maturation (22). The formation and expansion of coagulation results in hemostasis and the establishment of what will later shape the wound's extracellular matrix (38). This ECM exists essentially to encourage cell movement, adhesion, wound constriction and epithelialization. Transformation, organization and upkeep of the ECM rely upon various highly controlled intracellular and extracellular events. Enzyme-based events are very critical out of them as they are involved in the synthesis, supply and formation of counter biomolecules to combat and reverse the situation. This is necessary to normalize the typical physiological functions (39). There are different enzymes that are secreted exogenously and endogenously including collagenase, metalloproteinases, proteases, gelatinase, and tissue transglutaminase, which are all involved in healing and repair process $(21,40)$.

In wound restoration, mesenchymal cells discharge collagenase into the ECM and phagocytes lyse collagen, either acting on their surface or by functioning intracellularly. 


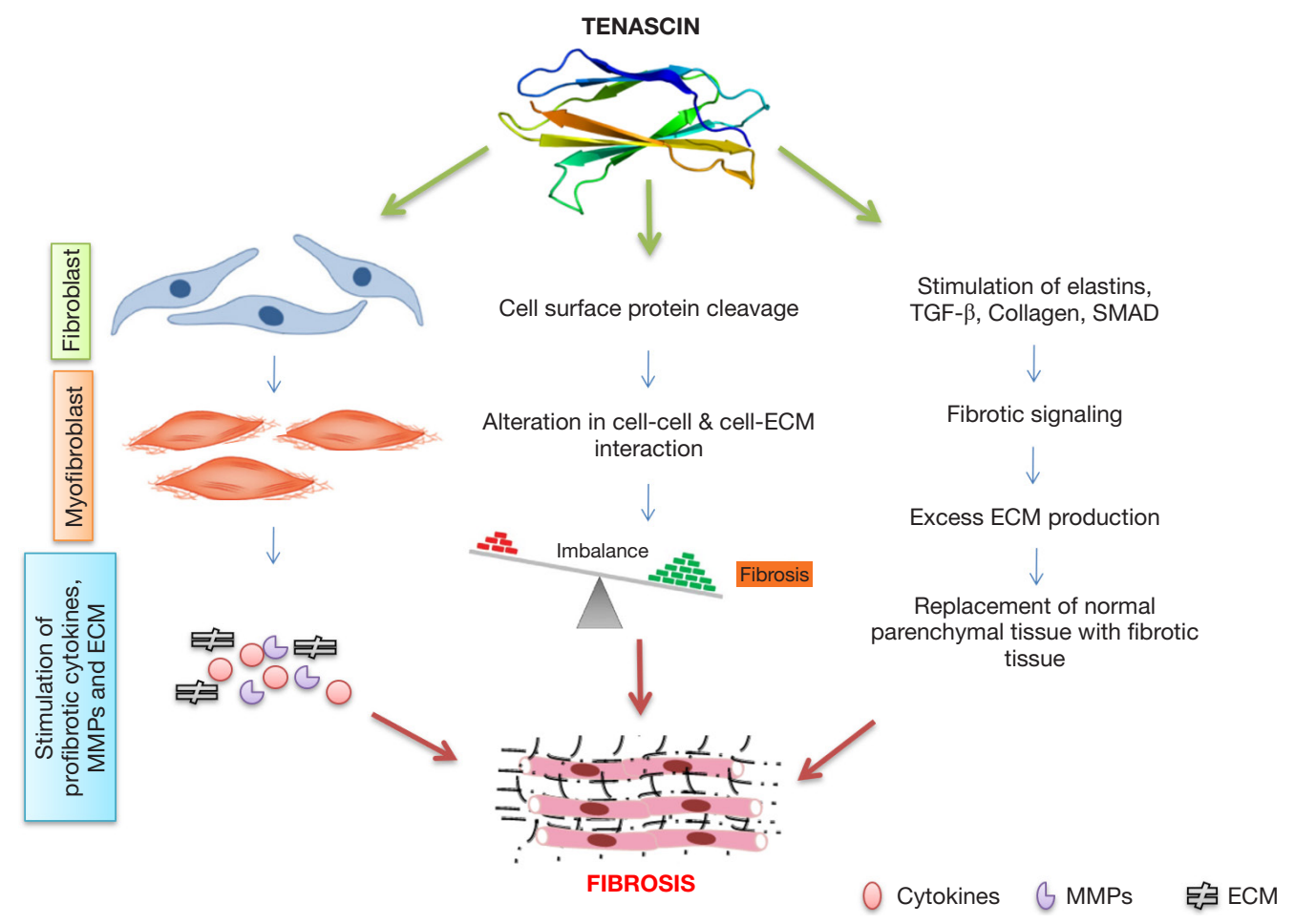

Figure 2 The role of tenascins in the pathogenesis of liver fibrosis. Tenascins significantly contribute to liver fibrosis by stimulating profibrogenic signaling and modulating functions of the extracellular matrix (ECM). MMPs, matrix metalloproteinases; SMAD, small mothers against decapentaplegic comprising a family of structurally similar proteins acting as main signal transducers for receptors of the TGF- $\beta$ superfamily; TGF- $\beta$, transforming growth factor- $\beta$.

Collagenase synthesis is triggered by IL-1, IL-6, PDGF, and others. The activated collagenases speed up the healing process by integrating the macrophage chemotaxis (41). Increased macrophage numbers stimulate the production of cytokines in the affected area fastening the healing mechanism. To reverse the collagenases activity some deciding factors exist in the body from which the strongest is $\alpha 2$-macroglobulin. But in fibrotic pathologies, excess synthesis of collagen occurs due to hyperactive collagenases, causing abnormal collagen deposition that consequently leads to liver fibrosis, rheumatoid arthritis, excess bone resorption subsequently followed by increased bone fracture probability and abnormal body architecture $(42,43)$.

Similarly, tissue transglutaminase, which is a protein cross-linking enzyme, is supposed to be involved in ECM repair mechanisms. It was found that wound induction by a UV source consisting $98.8 \%$ ultraviolet $\mathrm{A}$ and $1.2 \%$ ultraviolet $\mathrm{B}$ at ultraviolet doses of 60 to $120 \mathrm{~kJ}$ per m² resulted in increased activity of tissue transglutaminase as measured by incorporation of fluorescein-cadaverine into the ECM or by changes in the $\varepsilon$ ( $\gamma$-glutamyl) lysine crosslink. Exposure at higher doses $\left(180 \mathrm{~kJ}\right.$ per $\left.\mathrm{m}^{2}\right)$ of UV light resulted in 40-50\% cell death (44). These dying cells showed intense high levels of intracellular tissue transglutaminase as well as high quantities of this enzyme in surrounding ECM. However, further studies are warranted to understand how tissue transaminase contributes to repair and healing mechanisms (44). Beside enzymes, ROS play a crucial role in progression of injury and impeding the normal healing mechanism of the body. Superoxide dismutase (SOD) is a key antioxidant enzyme that eliminates ROS. Consequently, reduced SOD levels in the body attenuate healing and delaying the repair process (45).

\section{Reversal of fibrosis by targeting the extracellular matrix: novel targets and formulation-based approaches}

Fibrosis is an aberration in normal deposition of the ECM that transforms into a fibrotic scar. The deposition of 
these proteinaceous materials is regulated by proteases such as MMPs, TIMPs and LOXLs. Hence, targeting these enzymes or the deposition of the matrix proteins would prove to be the optimal strategy to reverse hepatic fibrosis. Integrins are the receptors mediating interactions between cells and their surrounding ECM. $\alpha v \beta 6$ and $\alpha v \beta 8$ integrins are proven to activate a latent form of TGF- $\beta 1$ that drives the entire fibrogenic cascade. $\alpha v \beta 3$ and $\alpha v \beta 5$ upregulates ECM-degrading proteases such as MMP2, -3 and -9 , that are involved in matrix degradation. Hence, targeting integrin would be an attractive target for the mitigation of liver fibrosis. The integrin-binding activity of proteins or peptides including the Arginine-glycine-aspartic acid tripeptide (RGD) motif with capacity to sequester integrin activity is also an attractive potential strategy for mitigation of liver fibrosis. In line, it has been shown that the administration of the RGD binding integrin antagonist led to apoptosis of HSCs and reduction in expression of the pro-inflammatory cytokine TGF- $\beta$ in a choline-deficient, amino-acid defined, high-fat diet (CDAHFD) mouse model of NASH (46).

Activated HSCs are the main source of aberrant ECM deposition. Hence, targeting the HSCs would be another good strategy to correct the abnormal changes within the ECM and to help to reverse hepatic fibrosis. The number of proteins highly expressed on the surface of activated HSCs can be an attractive strategy for targeted therapy in liver fibrosis. Type VI collagen receptor, retinol-binding protein (RBP) receptor, PDGF receptor, synaptophysin, insulin growth factor receptor II, low-density lipoprotein receptor and CD44 are few of the promising drug targets suitable to deliver nucleic acids or drugs to HSCs and to control the ECM production (47).

Cyclic RGD peptide-conjugated to human serum albumin in the molar ratio of 10:1 was shown to accumulate within the HSCs suggesting integrins as other good target structures for mitigation strategies (48). A similar strategy was used to deliver polymersome containing an anti-fibrotic agent within the HSCs. This inhibited the proliferation of HSCs and reduced the expression of $\alpha$-SMA and collagen as compared to the administration of polymersome given without conjugating it to the RGD peptide. Likewise, IFN$\alpha 1 b$ loaded liposomes showed a 10 -fold more efficient activity in the HSCs when conjugated to RGD peptide than unconjugated liposomes (49).

Quiescent HSCs (qHSCs) are the storage house for vitamin $\mathrm{A}$. It is estimated that around $80 \%$ of vitamin $\mathrm{A}$ in the form of retinyl palmitate is stored within the intracellular lipid droplets present in the HSCs. Interestingly, vitamin A is taken up more actively by activated HSCs than qHSCs. Vitamin A in the plasma is carried by RBP and then transported to the HSCs via the $\mathrm{RBP}$ receptor that is highly expressed in activated HSCs. Exploiting this basic physiology, gp46 siRNA was delivered to inhibit the production of collagen by silencing gp46 gene (50). Similarly, specific delivery of siRNA targeting HSP47 was achieved using vitamin A-coupled lipid nanoparticles (51). Likewise, a vitamin A terminallylinked to a complex copolymer enabled HSC-targeted delivery of miRNAs (52).

Glucose levels are closely related to the growth and proliferation of the liver tissue. Hyperglycemia and hyperinsulinemia induces the proliferation of HSCs leading to liver fibrosis. In line, inhibitors of dipeptidyl peptidase 4 (DPP4) such as Alogliptin were successfully proven to suppress the activation of HSCs. A recent study combining the effect of Alogliptin and the Takeda G protein coupled receptor 5 (TGR5) agonist oleanolic acid inducing the systemic release of glucagon like peptides from intestinal $\mathrm{L}$ cells and maintaining glycemic homeostasis, was proven to ameliorate liver fibrosis in diabetic rats (53).

DPP4 inhibition by Alogliptin alone also suppressed the activation of HSCs (54). Natural product based saponins of Rbizoma paridis showed down-regulation of ECM deposition and decreased the production of $\alpha$-SMA through suppressing the expression of VEGF, PDGF, and ERK1/2 (55). The long non-coding RNA HOTAIR, which induces activation of HSCs, is a target of miRNA124-3p (56). Rosiglitazone inhibits activation of HSCs by upregulating the expression of miRNA-124-3p (57). A recent study has proved that $\alpha$-calcitonin gene-related peptide is involved in cholangiocyte proliferation during cholestasis, thus silencing this gene seems to be beneficial in alleviating bile duct ligated liver fibrosis (58).

A recent study has shown that the Yes-associated protein (YAP) acting as a positive regulator of the $\mathrm{Wnt} / \beta$-catenin pathway, is overexpressed in fibrotic liver tissues. Thus, silencing of YAP ameliorated the disease by blocking TGF- $\beta$ regulated activation and proliferation in HSC-T6 cells (59). Extracellular matrix protein 1 (ECM1) is a recently discovered protein in the liver biopsy specimen of healthy and diseased patients, secreted by hepatocytes but not HSCs. It was observed that ECM1 expression was downregulated in fibrosis, while its depletion triggers the activation of TGF- $\beta 1$ (60). HSC-targeted combination of 
miRNA 29b and miRNA 122 regulates the profibrotic genes within the HSCs (52). Galectin 1 gene silencing results in HSCs apoptosis (61). Mir146a attenuates liver fibrosis by inhibiting TGF- $\beta 1$-mediated EMT (62). Similarly, PEGylated TRAIL treatment (63), nerve growth factor (NGF) (64) and gliotoxin (65) were reported to modulate HSC apoptosis, thereby controlling overexpression of pro fibrotic proteins.

ECM enzymes play a crucial role in regulating the accumulation and clearance of fibrotic tissue. Hence, recent studies have begun to focus on these enzymes for the reversal of liver fibrosis. Among these enzymes, LOXL enzymes have gained attention because of its role in crosslinking collagens. In two liver fibrosis models, carbon tetrachloride $\left(\mathrm{CCl}_{4}\right)$ or streptozotocin/ high fat diet-induced, PXS-5153A, a selective LOXL2/3 enzyme inhibitor reduced disease severity and improved liver function by diminishing collagen content and crosslinks (66). Transient overexpression of human MMP1 using an adenoviral delivery system has been shown to attenuate fibrosis by degrading collagen type I and type III, also stimulating the proliferation of hepatocytes (67). miR154 targeting MMP9 by regulating $\mathrm{Wnt} / \beta$-catenin pathway has also been shown to ameliorate liver fibrosis (68). TIMPs are another set of ECM enzymes that would be potential drug candidates. This was underpinned in a study using an antibody against TIMP-1 in rats that prevented activation of HSCs (69).

There have been a variety of experimental studies conducted targeting the ECM by different means to interfere with hepatic fibrosis. Chondroitin sulfate deoxycholic acid conjugates loaded with retinoic acid and doxorubicin were found accumulated within the Golgi apparatus and destroyed the production of collagen (70). Relaxin administration in well-established liver fibrosis model has shown to reduce collagen expression, $\alpha$-SMA levels, and lowering liver enzymes (71). However, being a peptide, several stability issues prevent its use in chronic treatment. To circumvent this problem, allosteric agonists have been developed for human relaxin receptor (RXFP1). Among them, ML290 is one such molecule that has been studied on fibrotic human liver organoids, human stellate cell lines LX-2 and also $\mathrm{CCl}_{4}$-induced liver fibrosis model. This strategy was effective in reducing fibrotic markers such as $\alpha$-SMA and also helped in controlling cell proliferation around the portal ducts, thus proving its potential as an anti-fibrotic agent (72). Mir-146a attenuates liver fibrosis by inhibiting TGF- $\beta 1$-mediated EMT (62). Halofuginone, an alkaloid extracted from Dichroa febrifuga inhibits TGF- $\beta$ induced phosphorylation of Smad3, representing a key player in the development of fibrogenesis (73).

Recent clinical trials have shown that diacerein, an anti-inflammatory drug, helps to improve liver fibrosis and steatosis in patients with type 2 diabetes and nonalcoholic fatty liver disease (NAFLD) (74) and atorvastatin reduced liver fibrosis in chronic hepatitis B patients (75). Another randomized clinical trial was recently initiated to study the effect of vitamin D supplementation on serum levels of vitamin D receptor (VDR), fibrogenic factors, and fibrogenic microRNAs in NAFLD patients (76). In a novel work, Sitanggang et al., co-cultured bone marrow derived stem cells (BMSCs) and HSCs. They showed that $\mathrm{CD} 90^{+} \mathrm{CD} 34^{-} \mathrm{BMSC}$ possess promising anti-liver fibrosis activity as evident from reduced ECM deposition and tenascin-C expression in the co-culture group (77). Table 1 enlists promising therapeutic targets and approaches for the mitigation of liver fibrosis. Table 2 contains a brief list of some of the important abbreviations used and their important functions.

\section{Conclusions and future directions}

Although our understanding of the basic pathophysiology of liver fibrosis has grown exponentially, the available management options are still limited. In this regard, the ECM proteins are a double edged sword. On one side, they are required for normal healing process, while on the other side their excessive secretion leads to severe complications in the pathogenesis of fibrosis. Several ECM proteins including LOX, MMPs, TIMPs, tenascins have emerged as plausible therapeutic targets to mitigate liver fibrosis. Further, different targeting formulations have been used to harness the physiological features of HSCs for the treatment of liver fibrosis. However, their efficacy was only successfully proven yet in experimental models of hepatic fibrosis. Future research activities in this area should be focused on pharmacodynamic studies in more preclinical animal models that better mimic relevant human clinical conditions. Further, the three-dimensional organoid systems can be of great value for understanding the matrix behavior within the boundaries of in vitro culture yet with closer resemblance to the real, in vivo physiological scenario. 
Table 1 Novel therapeutic targets and approaches utilized for the intervention of liver fibrosis

\begin{tabular}{|c|c|c|c|}
\hline Therapeutic target/approach & Animal model & Treatment outcome/parameters studied & References \\
\hline Integrins & $\begin{array}{l}\text { Choline-deficient, amino-acid defined, } \\
\text { high-fat diet (CDAHFD) mouse model of } \\
\text { NASH }\end{array}$ & $\begin{array}{l}\text { Apoptosis of HSCs and reduction in } \\
\text { expression of the pro-inflammatory } \\
\text { cytokine TGF- } \beta\end{array}$ & $(46)$ \\
\hline IFN- $\alpha 1$ b-loaded liposomes & HSCs & $\begin{array}{l}\text { Inhibited the proliferation of HSCs and } \\
\text { reduced expression of } \alpha \text {-SMA and } \\
\text { collagen }\end{array}$ & (49) \\
\hline $\begin{array}{l}\text { Dipeptidyl peptidase } 4 \text { (DPP4) } \\
\text { inhibitors }\end{array}$ & Diabetic rats and HSCs & $\begin{array}{l}\text { Ameliorated liver fibrosis in diabetic rats } \\
\text { and inhibited proliferation of HSCs }\end{array}$ & $(53,54)$ \\
\hline Rosiglitazone & $\begin{array}{l}\text { Male Otsuka long-evans tokushima fatty } \\
\text { (OLETF) rats and littermate long-evans } \\
\text { Tokushima Otsuka (LETO), HSCs and ob/ } \\
\text { ob mice }\end{array}$ & \multicolumn{2}{|l|}{$\begin{array}{l}\text { Inhibits activation of HSCs by upregulating (57) } \\
\text { expression of miRNA-124-3p }\end{array}$} \\
\hline $\begin{array}{l}\text { Yes-associated protein (YAP) } \\
\text { silencing }\end{array}$ & HSC-T6 cells & $\begin{array}{l}\text { Inhibited TGF- } \beta \text { regulated activation and } \\
\text { proliferation of HSCs }\end{array}$ & (59) \\
\hline $\begin{array}{l}\text { HSC-targeted combination of } \\
\text { miRNA-29b and miRNA-122 }\end{array}$ & HSCs & Regulates the profibrotic genes in HSCs & $(52)$ \\
\hline Galectin 1 gene silencing & $\mathrm{CCl}_{4}$-induced liver fibrosis & Apoptosis of HSCs & $(61)$ \\
\hline $\mathrm{miR}-146 \mathrm{a}$ & $\begin{array}{l}\text { Hepatocytes and } \mathrm{CCl}_{4} \text {-induced liver } \\
\text { fibrosis }\end{array}$ & Inhibits TGF- $\beta 1$-mediated EMT & (62) \\
\hline PEGylated TRAIL treatment & $\mathrm{CCl}_{4}$-induced liver fibrosis & $\begin{array}{l}\text { Induction of HSC apoptosis and control of } \\
\text { the overexpression of profibrotic proteins }\end{array}$ & $(63)$ \\
\hline Nerve growth factor (NGF) & $\begin{array}{l}\text { Human patient tissues of hepatitis } B \text { virus, } \\
\text { hepatitis } C \text { virus and non-viral hepatitis }\end{array}$ & $\begin{array}{l}\text { NGF was expressed at higher levels in } \\
\text { patients with hepatic fibrosis }\end{array}$ & (64) \\
\hline $\begin{array}{l}\text { Overexpression of human MMP1 } \\
\text { using adenoviral delivery system }\end{array}$ & Thioacetamide induced liver fibrosis & $\begin{array}{l}\text { Attenuates fibrosis by degrading type } \\
\text { I and type III collagen, stimulation of } \\
\text { hepatocytes }\end{array}$ & $(67)$ \\
\hline Antibody against TIMP-1 & $\mathrm{CCl}_{4}$-induced liver fibrosis & $\begin{array}{l}\text { Prevented activation of HSCs and reduced } \\
\text { the expression of } \alpha \text {-SMA }\end{array}$ & $(69)$ \\
\hline $\begin{array}{l}\text { Chondroitin sulfate deoxycholic acid } \\
\text { conjugates loaded with retinoic acid } \\
\text { and doxorubicin }\end{array}$ & $\begin{array}{l}\text { Activated HSCs, HepG2 cells and } \mathrm{CCl}_{4} \\
\text {-induced liver fibrosis }\end{array}$ & $\begin{array}{l}\text { Particles were found to accumulate in the } \\
\text { Golgi apparatus and suppressed collagen } \\
\text { synthesis }\end{array}$ & (70) \\
\hline $\begin{array}{l}\text { Relaxin and human relaxin receptor } \\
\text { (RXFP1) agonist ML290 }\end{array}$ & $\begin{array}{l}\text { Fibrotic human liver organoids, LX-2 cell } \\
\text { line and } \mathrm{CCl}_{4} \text {-induced liver fibrosis model }\end{array}$ & $\begin{array}{l}\text { Reduces the collagen expression, } \alpha-S M A \\
\text { levels, and lowering liver enzymes }\end{array}$ & $(71,72)$ \\
\hline Diacerein & Patients with type 2 diabetes and NAFLD & Improves liver fibrosis and steatosis & $(74)$ \\
\hline Atorvastatin & Chronic hepatitis B patients & Reduced liver fibrosis & $(75)$ \\
\hline $\begin{array}{l}\text { Bone marrow-derived stem cells } \\
\text { (BMSCs) }\end{array}$ & $\begin{array}{l}\text { Co-culture of bone marrow derived stem } \\
\text { cells and HSCs }\end{array}$ & $\begin{array}{l}\text { CD } 90^{+} \mathrm{CD} 34^{-} \text {BMSCs possess promising } \\
\text { anti-liver fibrosis activity }\end{array}$ & $(77)$ \\
\hline
\end{tabular}

$\alpha$-SMA, $\alpha$-smooth muscle actin; $\mathrm{CCl}_{4}$, carbon tetrachloride; HSC, hepatic stellate cells; IFN- $\alpha 1 \mathrm{~b}$, interferon- $\alpha 1 \mathrm{~b}$; MMP1, matrix metalloproteinase-1; NAFLD, non-alcoholic fatty liver disease; NASH, non-alcoholic steatohepatitis; TGF- $\beta$, transforming growth factor- $\beta$; TIMP-1, tissue inhibitor of metalloproteinases-1. 
Table 2 List of few selected molecules and their most important functions

\begin{tabular}{ll}
\hline $\begin{array}{l}\text { Abbreviation } \\
\text { Col-XIV }\end{array}$ & Full form and important role \\
DPP4 & Collagen-XIV, this collagen interacts with fibril surface and acts as a regulator of fibrillogenesis \\
E-cad & E-cadherin is an important epithelial protein marker whose expression declines during organ fibrosis \\
Hemopexin & Hemopexin (HPX) is a plasma protein mainly expressed in liver and is overexpressed during acute phase inflammation \\
HOTAIR & HOX transcript antisense RNA is overexpressed in metastatic cancers \\
NGF & Nerve growth factor is important factor involved in development and survival of neurons \\
RGD & Arginine-glycine-aspartic acid tripeptide, RGD is a cell recognition and attachment site for different extracellular matrix \\
& proteins \\
TGR5 & Takeda G protein coupled receptor 5 or G protein-coupled bile acid receptor 1 acts as cell surface receptor for bile acids \\
YAP & Yes-associated protein is a protein which functions as a transcriptional regulator
\end{tabular}

\section{Acknowledgments}

Funding: The laboratory of RW is supported by the German Research Foundation (SFB/TRR57) and the Interdisciplinary Centre for Clinical Research within the Faculty of Medicine at the RWTH Aachen University (IZKF Aachen, Project O3-1).

\section{Footnote}

Provenance and Peer Review: This article was commissioned by the Guest Editors (Ralf Weiskirchen and Wolfgang Stremmel) for the series "Unresolved Basic Issues in Hepatology" published in Annals of Translational Medicine. The article was sent for external peer review organized by the Guest Editors and the editorial office.

Peer Review File: Available at http://dx.doi.org/10.21037/ atm-20-2948

Conflicts of Interest: All authors have completed the ICMJE uniform disclosure form (available at http://dx.doi. org/10.21037/atm-20-2948). The series "Unresolved Basic Issues in Hepatology" was commissioned by the editorial office without any funding or sponsorship. RW served as the unpaid Guest Editor of the series and serves as an unpaid editorial board member of Annals of Translational Medicine from Aug 2020 to Jul 2022. The authors have no conflicts of interest to declare.

Ethical Statement: The authors are accountable for all aspects of the work in ensuring that questions related to the accuracy or integrity of any part of the work are appropriately investigated and resolved.

Open Access Statement: This is an Open Access article distributed in accordance with the Creative Commons Attribution-NonCommercial-NoDerivs 4.0 International License (CC BY-NC-ND 4.0), which permits the noncommercial replication and distribution of the article with the strict proviso that no changes or edits are made and the original work is properly cited (including links to both the formal publication through the relevant DOI and the license). See: https://creativecommons.org/licenses/by-nc-nd/4.0/.

\section{References}

1. Bataller R, Brenner DA. Liver fibrosis. J Clin Invest 2005;115:209-18.

2. Friedman SL. Liver fibrosis-from bench to bedside. J Hepatol 2003;38:S38-53.

3. Sarin SK,Maiwall R. Global burden of liver disease: a true burden on health sciences and economies. World Gastroenterol Organisation 2012;17:35-40.

4. Cotoi CG, Quaglia A. Normal Liver Anatomy and Introduction to Liver Histology. In: Textbook of Pediatric Gastroenterology, Hepatology and Nutrition. Springer, 2016:609-12.

5. Biagini G, Ballardini G. Liver fibrosis and extracellular matrix. J Hepatol 1989;8:115-24.

6. Arriazu E, Ruiz de Galarreta M, Cubero FJ, et al. Extracellular matrix and liver disease. Antioxid Redox 
Signal 2014;21:1078-97.

7. Frantz C, Stewart KM, Weaver VM. The extracellular matrix at a glance. J Cell Sci 2010;123: 4195-200.

8. Arenson DM, Friedman SL, Bissel DM. Formation of extracellular matrix in normal rat liver: lipocytes as a major source of proteoglycan. Gastroenterology 1988;95:441-7.

9. Krishnan A, Li X, Kao WY, et al. Lumican, an extracellular matrix proteoglycan, is a novel requisite for hepatic fibrosis. Lab Invest 2012;92:1712-25.

10. Bedossa P, Paradis V. Liver extracellular matrix in health and disease. J Pathol 2003;200:504-15.

11. Dallas SL, Sivakumar P, Jones CJ, et al. Fibronectin regulates latent transforming growth factor- $\beta$ (TGF $\beta$ ) by controlling matrix assembly of latent TGF $\beta$-binding protein-1. J Biol Chem 2005;280:18871-80.

12. Aziz-Seible RS, Casey CA. Fibronectin: functional character and role in alcoholic liver disease. World J Gastroenterol 2011;17:2482-99.

13. Koukoulis GK, Shen J, Virtanen I, et al. Vitronectin in the cirrhotic liver: an immunomarker of mature fibrosis. Hum Pathol 2001;32:1356-62.

14. Clément B, Rescan PY, Baffet G, et al. Hepatocytes may produce laminin in fibrotic liver and in primary culture. Hepatology 1988;8:794-803.

15. Regős E, Karászi K, Reszegi A, et al. Syndecan-1 in liver diseases. Pathol Oncol Res 2020;26:813-9.

16. Baghy K, Iozzo RV, Kovalszky I. Decorin-TGF- $\beta$ axis in hepatic fibrosis and cirrhosis. J Histochem Cytochem 2012;60:262-8.

17. Hernandez-Gea V, Friedman SL. Pathogenesis of liver fibrosis. Annu Rev Pathol 2011;6:425-56.

18. Lee SJ, Yoo JD, Choi SY, et al. The expression and secretion of vimentin in the progression of non-alcoholic steatohepatitis. BMB Rep 2014;47:457-62.

19. Arthur MJ. Degradation of matrix proteins in liver fibrosis. Pathol Res Pract 1994;190:825-33.

20. Arthur MJ. Fibrogenesis II. Metalloproteinases and their inhibitors in liver fibrosis. Am J Physiol Gastrointest Liver Physiol 2000;279:G245-9.

21. Duarte S, Baber J, Fujii T, et al. Matrix metalloproteinases in liver injury, repair and fibrosis. Matrix Biol 2015;4446:147-56.

22. Armstrong DG, Jude EB. The role of matrix metalloproteinases in wound healing. J Am Podiatr Med Assoc 2002;92:12-8.

23. Kim Y, Ko H, Kwon IK, et al. Extracellular matrix revisited: roles in tissue engineering. Int Neurourol J 2016;20:S23-9.
24. Ikenaga N, Peng ZW, Vaid KA, et al. Selective targeting of lysyl oxidase-like 2 (LOXL2) suppresses hepatic fibrosis progression and accelerates its reversal. Gut 2017;66:1697-708.

25. Midwood KS, Chiquet M, Tucker RP, et al. Tenascin-C at a glance. J Cell Sci 2016;129:4321-7.

26. Frantz C, Stewart KM, Weaver VM. The extracellular matrix at a glance. J Cell Sci 2010;123:4195-200.

27. Midwood KS, Orend G. The role of tenascin-C in tissue injury and tumorigenesis. J Cell Commun Signal 2009;3:287-310.

28. Midwood KS, Hussenet T, Langlois B, et al. Advances in tenascin-C biology. Cell Mol Life Sci 2011;68:3175-99.

29. Jones PL, Jones FS. Tenascin-C in development and disease: gene regulation and cell function. Matrix Biol 2000;19:581-96.

30. Kasprzycka M, Hammarstrom C, Haraldsen G. Tenascins in fibrotic disorders-from bench to bedside. Cell Adh Migr 2015;9:83-9.

31. Arthur MJ, Mann DA, Iredale JP. Tissue inhibitors of metalloproteinases, hepatic stellate cells and liver fibrosis. J Gastroenterol Hepatol 1998;13 Suppl:S33-8.

32. Ramadori G, Schögler S, Veit T, et al. Tenascin gene expression in rat liver and in rat liver cells. In vivo and in vitro studies. Virchows Arch B Cell Pathol Incl Mol Pathol 1991;60:145-53.

33. Baiocchini A, Montaldo C, Conigliaro A, et al. Extracellular matrix molecular remodeling in human liver fibrosis evolution. PLOS ONE 2016;11:e0151736.

34. Miyazaki H, Van Eyken P, Roskams T, et al. Transient expression of tenascin in experimentally induced cholestatic fibrosis in rat liver: an immunohistochemical study. J Hepatol 1993;19:353-66.

35. Trebaul A, Chan EK, Midwood KS. Regulation of fibroblast migration by tenascin-C. Biochem Soc Trans 2007;35:695-7.

36. Brellier F, Hostettler K, Hotz HR, et al. Tenascin-C triggers fibrin accumulation by downregulation of tissue plasminogen activator. FEBS Letters 2011;585:913-20.

37. El-Karef A, Kaito M, Tanaka H, et al. Expression of large tenascin-C splice variants by hepatic stellate cells/myofibroblasts in chronic hepatitis C. J Hepatol 2007;46:664-73.

38. Hatz RA, von Jan NCS, Schildberg FW. The role of collagenase in wound healing. In: Proteolytic enzymes and wound healing. Berlin, Heidelberg: Springer Berlin Heidelberg, 1994.

39. Mignatti P, Welgus HG, Rifkin DB. Role of degradative 
enzymes in wound Healing. In: Clark RAF, Henson PM. editors. The Molecular and cellular biology of wound repair. Boston, MA: Springer, 1988:497-523.

40. Sinclair RD, Ryan TJ. Proteolytic enzymes in wound healing: the role of enzymatic debridement. Australas J Dermatol 1994;35:35-41.

41. Pilcher BK, Wang M, Qin XJ, et al. Role of matrix metalloproteinases and their inhibition in cutaneous wound healing and allergic contact hypersensitivity. Ann N Y Acad Sci 1999;878:12-24.

42. Parks WC. Matrix metalloproteinases in repair. Wound Repair Regen 1999;7:423-32.

43. McCarty SM, Percival SL. Proteases and Delayed Wound Healing. Adv Wound Care (New Rochelle) 2013;2:438-47.

44. Gross SR, Balklave Z, Griffin M. Importance of tissue transglutaminase in repair of extracellular matrices and cell death of dermal fibroblasts after exposure to a solarium ultraviolet A source. J Invest Dermatol 2003;121:412-23.

45. Fujiwara T, Duscher D, Rustad KC, et al. Extracellular superoxide dismutase deficiency impairs wound healing in advanced age by reducing neovascularization and fibroblast function. Exp Dermatol 2016;25:206-11.

46. Ulmasov B, Noritake H, Carmichael P, et al. An inhibitor of arginine-glycine-aspartate-binding integrins reverses fibrosis in a mouse model of nonalcoholic steatohepatitis. Hepatol Commun 2018;3:246-61.

47. Chen Z, Jain A, Liu H, et al. Targeted drug delivery to hepatic stellate cells for the treatment of liver fibrosis. J Pharmacol Exp Ther 2019;370:695-702.

48. Beljaars L, Molema G, Schuppan D, et al. Successful targeting to rat hepatic stellate cells using albumin modified with cyclic peptides that recognize the collagen type VI receptor. J Biol Chem 2000;275:12743-51.

49. Yang J, Hou Y, Ji G, et al. Targeted delivery of the RGD-labeled biodegradable polymersomes loaded with the hydrophilic drug oxymatrine on cultured hepatic stellate cells and liver fibrosis in rats. Eur J Pharm Sci 2014;52:180-90.

50. Sato Y, Murase K, Kato J, et al. Resolution of liver cirrhosis using vitamin A-coupled liposomes to deliver siRNA against a collagen-specific chaperone. Nat Biotechnol 2008;26:431-42.

51. Poilil Surendran S, Thomas RG, Moon MJ, et al. Nanoparticles for the treatment of liver fibrosis. Int J Nanomedicine 2017;12:6997-7006.

52. Wu J, Huang J, Kuang S, et al. Synergistic MicroRNATherapy in liver fibrotic rat using MRI-visible nanocarrier targeting hepatic stellate cells. Adv Sci (Weinh)
2019;6:1801809.

53. Kaya D, Kaji K, Tsuji Y, et al. TGR5 activation modulates an inhibitory effect on liver fibrosis development mediated by anagliptin in diabetic rats. Cells 2019;8:1153.

54. Zhang H, Sun D, Wang G, et al. Alogliptin alleviates liver fibrosis via suppression of activated hepatic stellate cell. Biochem Biophys Res Commun 2019;511:387-93.

55. Hong Y, Han YQ, Wang YZ, et al. Paridis Rhizoma Sapoinins attenuates liver fibrosis in rats by regulating the expression of RASAL1/ERK1/2 signal pathway. J Ethnopharmacol 2016;192:114-22.

56. Yu F, Chen B, Dong P, et al. HOTAIR epigenetically modulates PTEN expression via MicroRNA-29b: a novel mechanism in regulation of liver fibrosis. Mol Ther 2017;25:205-17.

57. Zhi SC, Chen SZ, Li YY, et al. Rosiglitazone inhibits activation of hepatic stellate cells via up-regulating microRNA-124-3p to alleviate hepatic fibrosis. Dig Dis Sci 2019;64:1560-70.

58. Wan Y, Ceci L, Wu N, et al. Knockout of $\alpha$-calcitonin gene-related peptide attenuates cholestatic liver injury by differentially regulating cellular senescence of hepatic stellate cells and cholangiocytes. Lab Invest 2019;99:764-76.

59. Yu HX, Yao Y, Bu FT, et al. Blockade of YAP alleviates hepatic fibrosis through accelerating apoptosis and reversion of activated hepatic stellate cells. Mol Immunol 2019;107:29-40.

60. Dooley S, Fan W, Hammad S, et al. ECM1 is a gatekeeper that restrains TGF- $\beta$ activation to maintain liver homeostasis and prevent fibrogenesis. Z Gastroenterol, 2019;57:P1.10.

61. Jiang ZJ, Shen QH, Chen YH, et al. Galectin-1 gene silencing inhibits the activation and proliferation but induces the apoptosis of hepatic stellate cells from mice with liver fibrosis. Int J Mol Med 2019;43:103-16.

62. Zou Y, Li S, Li Z, et al. MiR-146a attenuates liver fibrosis by inhibiting transforming growth factor- $\beta 1$ mediated epithelial-mesenchymal transition in hepatocytes. Cell Signal 2019;58:1-8.

63. Oh Y, Park O, Swierczewska M, et al. Systemic PEGylated TRAIL treatment ameliorates liver cirrhosis in rats by eliminating activated hepatic stellate cells. Hepatology 2016;64:209-23.

64. Amoras ES, Gomes ST, Freitas FB, et al. NGF and P75NTR gene expression is associated with the hepatic fibrosis stage due to viral and non-viral causes. PLoS One 2015;10:e0121754.

65. Wright MC, Issa R, Smart DE, et al. Gliotoxin stimulates 
the apoptosis of human and rat hepatic stellate cells and enhances the resolution of liver fibrosis in rats. Gastroenterology 2001;121:685-98.

66. Schilter H, Findaly AD, Perryman L, et al. The lysyl oxidase like 2/3 enzymatic inhibitor, PXS-5153A, reduces crosslinks and ameliorates fibrosis. J Cell Mol Med 2019;23:1759-70.

67. Iimuro Y, Nishio T, Morimoto T, et al. Delivery of matrix metalloproteinase-1 attenuates established liver fibrosis in the rat. Gastroenterology 2003;124:445-58.

68. Shi G., Molecular mechanism of miR-154 targeting MMP-9 by regulating $W \mathrm{nt} / \beta$-catenin pathway in liver fibrosis. Int J Clin Exp Pathol 2017;10:8839-44.

69. Parsons CJ, Bradford BU, Pan CQ, et al. Antifibrotic effects of a tissue inhibitor of metalloproteinase-1 antibody on established liver fibrosis in rats. Hepatology 2004;40:1106-15.

70. Luo J, Zhang P, Zhao T, et al. Golgi apparatus-targeted chondroitin-modified nanomicelles suppress hepatic stellate cell activation for the management of liver fibrosis. ACS Nano 2019;13:3910-23.

71. Bennett RG, Heimann Dg, Tuma DJ. Relaxin reduces fibrosis in models of progressive and established hepatic fibrosis. Ann NY Acad Sci 2009;1160:348-9.

72. Kaftanovskaya E, Ng HH, Soula M, et al. Small molecule allosteric agonist of relaxin receptor ML290 demonstrates

Cite this article as: Khurana A, Sayed N, Allawadhi P, Weiskirchen R. It's all about the spaces between cells: role of extracellular matrix in liver fibrosis. Ann Transl Med 2021;9(8):728. doi: 10.21037/atm-20-2948 antifibrotic properties in liver fibrosis. 21st European Congress of Endocrinology. Endocrine Abstracts 2019;63:GP223.

73. Karakoyun B. The promising role of anti-fibrotic agent halofuginone in liver fibrosis/cirrhosis. In: Tsoulfas G. editor. Liver cirrhosis: update and current challenges. 2017:257.

74. Leite NC, Viegas BB, Villela-Nogueira CA, et al. Efficacy of diacerein in reducing liver steatosis and fibrosis in patients with type 2 diabetes and non-alcoholic fatty liver disease: A randomized, placebo-controlled trial. Diabetes Obes Metab 2019;21:1266-70.

75. Sigler MA, Congdon L, Edward KL. An evidencebased review of statin use in patients with nonalcoholic fatty liver disease. Clin Med Insights Gastroenterol 2018;11:1179552218787502.

76. Ebrahimpour-Koujan S, Sohrabpour AA, Foroughi F, et al. Effects of vitamin D supplementation on liver fibrogenic factors in non-alcoholic fatty liver patients with steatohepatitis: study protocol for a randomized clinical trial. Trials 2019;20:153.

77. Sitanggang EJ, Antarianto RD, Jusman SWA, et al., Bone marrow stem cells anti-liver fibrosis potency: inhibition of hepatic stellate cells activity and extracellular matrix deposition. Int J Stem cells 2017;10:69-75. 